\title{
ON BOUNDS FOR THE DERIVATIVE OF ANALYTIC FUNCTIONS
}

\author{
DOROTHY BROWNE SHAFFER
}

\begin{abstract}
Let $g(z)$ be analytic and $|g(z)| \leqq 1$ in $|z|<1 ; g(z)=$ $\sum_{k=p}^{\infty} a_{k} z^{k}, p \geqq 1$, then a sharp upper bound is derived for $\left|g^{\prime}(z)\right|$. Let $h(z)$ be analytic for $|z|<1, h(0)=1, \operatorname{Re} h(z)>\alpha$ where $0 \leqq \alpha<1$, then bounds for $\left|h^{\prime}(z)\right|$ are derived and sharpened for a function with missing terms.
\end{abstract}

In this paper distortion theorems are established for the derivative of analytic functions of two types; first, functions bounded by the constant one in the unit disc, and secondly for functions whose real part is bounded from below.

In the literature frequent use is made of known upper bounds for the modulus of the derivative of an analytic function bounded in the unit disc. The first theorem in this paper establishes a more powerful inequality based on the additional assumption that the function vanishes at the origin. In the consequent theorems this result is applied to functions which assume values in a half plane. An estimate due to Goluzin [3, p. 290] yields the inequality

$$
\left|f^{\prime}(z)\right| \leqq p|z|^{p-1}\left(1-|f(z)|^{2}\right) /\left(1-|z|^{2 p}\right)
$$

for a function $f(z)$, regular and $|f(z)| \leqq 1$ in $|z|<1$ with expansion $f(z)=$ $c_{0}+\sum_{k=p}^{\infty} c_{k} z^{k}, p \geqq 1$. However this bound will not be assumed for a function vanishing at the origin and it is the object of the first theorem to obtain an improved estimate for functions with $c_{0}=0$.

THEOREM 1. For $|z|<1$, let $g(z)$ be regular, $|g(z)| \leqq 1$ and have the expansion $g(z)=\sum_{k=p}^{\infty} a_{k} z^{p}, p \geqq 1 ;$ then

(1a) $\left|g^{\prime}(z)\right| \leqq p|z|^{p-1}$ for $|z| \leqq\left(\left[1+p^{2}\right]^{1 / 2}-1\right) / p$,

$$
\begin{aligned}
& \left|g^{\prime}(z)\right| \leqq|z|^{p-2}\left[4|z|^{2}+p^{2}\left(1-|z|^{2}\right)^{2}\right] / 4\left(1-|z|^{2}\right) \\
& \text { for }|z|>\left(\left[1+p^{2}\right]^{1 / 2}-1\right) / p .
\end{aligned}
$$

Proof. It follows from the maximum modulus theorem that for a function $g(z)$ defined as in the hypothesis of our theorem we have

Presented to the Society, January 18, 1972; received by the editors February 21, 1972 and, in revised form, April 27, 1972.

AMS (MOS) subject classifications (1969). Primary 3010.

(c) American Mathematical Society 1973 
$|g(z)| \leqq|z|^{p}$. Let

$$
g(z)=f(z) z^{p-1}
$$

then $f(0)=0,|f(z)| \leqq|z|$.

The function $f(z)$ can be written

$$
\left.\left[f\left(z_{0}\right)-f(z)\right] /\left(z_{0}-z\right)=\left[1-\overline{f\left(z_{0}\right.}\right) f(z)\right] \phi(z) /\left(1-\bar{z}_{0} z\right)
$$

where $|\phi(z)| \leqq 1$. The condition $f(0)=0 \Rightarrow f\left(z_{0}\right)=z_{0} \phi(0)$. Using the notation $|\phi(0)|=a$, we obtain $[\mathbf{1}$, p. 19]:

$$
\left|\phi\left(z_{0}\right)\right| \leqq\left(a+\left|z_{0}\right|\right) /\left(1+a\left|z_{0}\right|\right) .
$$

After simplification the following inequality is obtained by differentiating (2), substituting in the result the bounds obtained by putting $z=z_{0}$ in (3) and (4):

$$
\left(1-|z|^{2}\right)\left|g^{\prime}(z)\right| /|z|^{p-1} \leqq-a^{2}|z|+a p\left(1-|z|^{2}\right)+|z| .
$$

The maximum of the right-hand side is assumed for $a=p\left(1-|z|^{2}\right) / 2|z|$. The requirement $a \leqq 1$ is met for $|z| \geqq\left(\left[1+p^{2}\right]^{1 / 2}-1\right) / p$ which yields the estimate (lb). Case (la) follows for $a=1$.

For $p=1$ the Goluzin inequality reduces to the well-known bound for the absolute value of the derivative, and Theorem 1 reduces to the known improvement due to Dieudonné [1, p. 19].

In Theorem 1 equality in (la) is obtained for the function $f(z)=e^{i \theta} z$, $g(z)=e^{i \theta} z^{p}$. For bound (1b), the extremal function must have the form $g(z)=e^{i \theta} z^{p}(z+b) /(1+b z)$. For simplicity $b$ may be chosen real, and equality is obtained for real $z_{0}>0$, and $\left|z_{0}\right|>\left[\left(1+p^{2}\right)^{1 / 2}-1\right] p^{-1}$, if $\left(b+z_{0}\right) /\left(1+b z_{0}\right)=p\left(1-z_{0}^{2}\right) / 2 z_{0}$, or $b=\left[p-(2+p) z_{0}^{2}\right] /\left(2-p+p z_{0}^{2}\right) z_{0}$.

The rest of this paper deals with applications to functions which assume values in a half plane.

THEOREM 2. Let $h(z)$ be analytic for $|z|<1$ and $\operatorname{Re} h(z)>\alpha, 0 \leqq \alpha<1$, and $h(z)$ has the expansion $h(z)=1+a_{p} z^{p}+a_{p+1} z^{p+1}+\cdots, p \geqq 1$. Then

(5a) $\left|h^{\prime}(z)\right| \leqq \frac{p|z|^{p-1}|h(z)+c|^{2}}{c+1}$ for $|z| \leqq \frac{\left[1+p^{2}\right]^{1 / 2}-1}{p}$,

$$
\left|h^{\prime}(z)\right| \leqq \frac{|z|^{p-2}\left[4|z|^{2}+p^{2}\left(1-|z|^{2}\right)^{2}\right]|h(z)+c|^{2}}{4\left(1-|z|^{2}\right)(1+c)}
$$

where $c=1-2 \alpha$.

$$
\text { for }|z|>\left(\left[1+p^{2}\right]^{1 / 2}-1\right) / p
$$

Proof. Let $g(z)=(h(z)-1) /(h(z)+c)=b_{1} z^{p}+\cdots$, then $g(z)$ is analytic for $|z|<1,|g(z)| \leqq|z|^{p},\left|h^{\prime}(z)\right|=\left|g^{\prime}(z)\right||h(z)+c|^{2} /(c+1)$. Applying the estimate of the previous theorem for $\left|g^{\prime}(z)\right|$, we obtain bounds (5a) and (5b). 
In Theorem 2 the extremal functions $h(z)$ correspond to the extremal functions $g(z)$ of Theorem 1. In Theorem 2, $p=1$, the functions $h(z)=$ $(1+c z) /(1-z)$ and $h(z)=\left(1+b(1+c) z+c z^{2}\right) /\left(1-z^{2}\right)$ will have derivatives assuming the upper bounds (5a) and (5b). The function $h(z)=$ $\left(1+c z^{p}\right) /\left(1-z^{p}\right)$ is extremal for arbitrary $p$.

Estimates for the upper bounds of $\left|h^{\prime}(z)\right|$ were obtained by Tonti and Trahan [5]. These were derived on the same assumptions as this paper; being based on the standard inequalities for the derivative of bounded function, the results were less sharp.

In the following theorems estimates are derived for the derivative $h^{\prime}(z)$ independent of the value of the function $h(z)$.

THEOREM 3. Let the function $h(z)$ be the same as in Theorem $2, p=1$, then

$$
\left|h^{\prime}(z)\right| \leqq(c+1) /(1-|z|)^{2} \text { for }|z|<1 .
$$

Proof. With the notation of the proof in Theorem 2 we obtain $h(z)=$ $(1+c g(z)) /[1-g(z)]$ where $g(z)$ is subordinate to $z$. By calculations similar to that of Theorem 1 the following estimate is found to hold:

$$
\left|h^{\prime}(z)\right| \leqq(1+c)(a+|z|) /\left(1-|z|^{2}\right)(1-a|z|) .
$$

This is a monotonically increasing function of $a ;(6)$ is obtained by substitution of $a=1$.

THEOREM 4. With the same hypothesis as in Theorem 2, $p=1$, and Theorem 3,

$$
\left|h^{\prime}(z) / h(z)\right| \leqq(1+c) /(1-|z|)(1+c|z|) \text { for }|z|<1 .
$$

In the proof $h^{\prime}(z) / h(z)$ is calculated in terms of $g^{\prime}(z)$ and $g(z)$. With the previous notation we obtain

$$
\left|h^{\prime}(z) / h(z)\right|=(c+1)(a+|z|) /\left(1-|z|^{2}\right)(1+c a|z|) .
$$

The result of the theorem follows for $a=1$.

Theorem 4 reduces to the result of MacGregor [4] for a function with positive real part, $c=1$ and to the estimate obtained by Causey and Merkes [2] for $\alpha=\frac{1}{2}$ or $c=0$.

The last two theorems refer again to the special case of the function $h(z)$ with missing terms.

THEOREM 5. Let $h(z)$ be as in Theorem 2, then

$$
\left|h^{\prime}(z)\right| \leqq p|z|^{p-1}(1+c) /\left(1-|z|^{p}\right)^{2} \text { for }|z| \leqq\left(\left[1+p^{2}\right]^{1 / 2}-1\right) / p .
$$


The proof depends on combining the inequality

$$
|h(z)+c|=(1+c) /|1-g(z)| \leqq(1+c) /\left[1-|z|^{p}\right]
$$

with the result (5a) of Theorem 2. By the same method we also obtain

THEOREM 6. For the function $h(z)$ of Theorem 2 the following estimate holds

$$
\begin{aligned}
& \left|h^{\prime}(z) / h(z)\right| \leqq p|z|^{p-1}(1+c) /\left(1-|z|^{p}\right)\left(1+c|z|^{p}\right) \\
& \quad \text { for }|z| \leqq\left(\left[1+p^{2}\right]^{1 / 2}-1\right) / p .
\end{aligned}
$$

The results of the previous theorems can be applied to functions $f(z)=z+a_{2} z^{2}+\cdots$ in $|z|<1$ with $\operatorname{Re} f^{\prime}(z)>\alpha$. Estimates for $f^{\prime \prime}(z)$ are obtained by identifying $h(z)=f^{\prime}(z)$. For example we obtain $\left|f^{\prime \prime}(z)\right| f(z) \mid \leqq$ $(c+1) /(1-|z|)(1+c|z|)$ which leads to a radius of convexity $|z| \leqq$ $\left(-1+[1+c]^{1 / 2}\right) / c, c>0$, for functions of this class. However this estimate is sharp only for $c=1$ [4].

\section{BIBLIOGRAPHY}

1. C. Carathéodory, Theory of functions of a complex variable. Vol. 2, Chelsea, New York, 1954. MR 16, 346.

2. W. M. Causey and E. P. Merkes, Radii of starlikeness of certain classes of analytic functions. J. Math. Anal. Appl. 31 (1970), 579-586. MR 41 \#8644.

3. G. M. Goluzin, Geometric theory of functions of a complex variable, GITTL, Moscow, 1952; German transl., VEB Deutscher Verlag, Berlin, 1957; English transl., Transl. Math. Monographs, vol. 26, Amer. Math. Soc., Providence, R.I., 1969. MR 15, 112; MR 19, 735; MR 40 \#308.

4. T. H. MacGregor, Functions whose derivative has a positive real part, Trans. Amer. Math. Soc. 104 (1962), 532-537. MR 25 \#4090.

5. N. E. Tonti and D. H. Trahan, Analytic functions whose real parts are bounded below, Math. Z. 115 (1970), 252-258. MR 41 \#7116.

Courant Institute of Mathematical Sciences, New York University, New YORK, NEW YORK 10012 06430

Department of Mathematics, Fairfield University, Fairfield, Connecticut 